\title{
Generalized Out-of-Plane Equilibrium Points in the Frame of Elliptic Restricted Three-Body Problem: Impact of Oblate Primary and Luminous-Triaxial Secondary
}

\author{
Aminu Abubakar Hussain (iD) ${ }^{1}$ and Aishetu Umar $\mathbb{D D}^{2}$ \\ ${ }^{1}$ Department of Mathematics, Faculty of Natural and Applied Sciences, Nasarawa State University, Keffi, Nigeria \\ ${ }^{2}$ Department of Mathematics, Faculty of Physical Science, Ahmadu Bello University, Zaria, Nigeria \\ Correspondence should be addressed to Aminu Abubakar Hussain; dadinduniya@gmail.com
}

Received 29 October 2018; Accepted 20 January 2019; Published 26 February 2019

Academic Editor: Michael Kueppers

Copyright ( 2019 Aminu Abubakar Hussain and Aishetu Umar. This is an open access article distributed under the Creative Commons Attribution License, which permits unrestricted use, distribution, and reproduction in any medium, provided the original work is properly cited.

\begin{abstract}
This paper studies the motion of a third body near the $1^{\text {st }}$ family of the out-of-plane equilibrium points, $L_{6,7}$, in the elliptic restricted problem of three bodies under an oblate primary and a radiating-triaxial secondary. It is seen that the pair of points $\left(\xi_{0}, 0, \pm \zeta_{0}\right)$ which correspond to the positions of the $1^{\text {st }}$ family of the out-of-plane equilibrium points, $L_{6,7}$, are affected by the oblateness of the primary, radiation pressure and triaxiality of the secondary, semimajor axis, and eccentricity of the orbits of the principal bodies. But the point $\pm \zeta_{0}$ is unaffected by the semimajor axis and eccentricity of the orbits of the principal bodies. The effects of the parameters involved in this problem are shown on the topologies of the zero-velocity curves for the binary systems PSR 1903+0327 and DPLeonis. An investigation of the stability of the out-of-plane equilibrium points, $L_{6,7}$ numerically, shows that they can be stable for $0.32 \leq \mu \leq 0.5$ and for very low eccentricity. $L_{6,7}$ of PSR $1903+0327$ and DP-Leonis are however linearly unstable.
\end{abstract}

\section{Introduction}

Generally, the motion of a body is influenced by the mutual gravitational attraction from other bodies. In a situation where we consider an isolated dynamical system of three gravitational interacting bodies $m_{1}, m_{2}$, and $m_{3}$, and where the third body $m_{3}$ (called the infinitesimal mass) has a very small mass compared to the other bodies $m_{1}$ and $m_{2}$ (called the principal bodies, where $m_{1} \geq m_{2}$ ), the third body $m_{3}$ has a very small effect (negligible) on the motion of the principal bodies $m_{1}$ and $m_{2}$ (i.e., $m_{3} \ll m_{1} \& m_{2}$ ). These principal bodies called the primaries (i.e., $m_{1}$ is called bigger primary or primary and $m_{2}$ is called smaller primary or secondary, respectively) act as a pair, which has their motion approximated by a two-body problem with their trajectories predetermined. The motion of the third body as affected by the principal bodies is the subject of the restricted three-body problem (R3BP). This is what made the R3BP a more tractable case than the general three-body problem. If the principal bodies are restricted to a circular or elliptic orbit while the third body of infinitesimal mass orbits in the plane of motion established by the principal bodies, then we, respectively, have circular or elliptic R3BP (CR3BP or ER3BP). See [1].

Over decades, the study of the existence of some families of particular solutions in both circular and elliptic R3BP has received the attention of various researchers like [1-13] among others.

Reference [14] was the first to point out the existence of the out-of-plane equilibrium points in the cases of SunPlanet-Particle and Galaxy Kernel-Sun-Particle and he found the two equilibrium points $L_{6,7}$ on the $x-z$ plane symmetrically with respect to the orbital plane. The case where the Poynting-Robertson effect is taken into account was treated by [15].

Reference [16] proved the existence of a second family of the out-of-plane equilibrium points $L_{8,9}$ when the principal bodies are both luminous under certain condition (i.e., the relation between the mass and radiation pressure parameters). They also studied the stability for $\mu=0.5$ and $\mu=$ 0.2 . 
The periodic solution about the out-of-plane equilibrium points in the photogravitational restricted three-body problem was carried out by [17]. They found that two such families exist: the first family originates and terminates on the same equilibrium point, while the second family terminates by flattening on the orbital plane.

Afterwards, various studies of the out-of-plane equilibrium points have been carried out by other researchers like [18-23] and many more.

Reference [5] studied the motion in the photogravitational elliptic restricted three-body problem under an oblate primary. In their study, they found that the out-of-plane equilibrium points are unstable for any combination of the parameters under their own consideration. Also, [24] studied the family of out-of-plane equilibrium points $L_{6,7}$ in the elliptic restricted three-body problem with radiating and oblate primaries. They concluded that the positions and stability depend on the oblateness and radiation pressure of the primaries and the eccentricity of their orbits.

Reference [25] examined the out-of-plane equilibrium points in the photogravitational CR3BP with oblateness and $\mathrm{P}-\mathrm{R}$ drag. In their numerical study, they found that the out-of-plane equilibrium points are unstable in the sense of Lyapunov due to the presence of positive real root.

Recently, [26] investigated the influence of PoyntingRobertson drag and oblateness on the existence and stability of the out-of-plane equilibrium points in the spatial elliptic restricted three-body problem. They also found $L_{6,7}$ to be unstable. Reference [27] studied the Newton-Raphson basins of convergence of the out-of-plane equilibrium points in the Copenhagen problem with oblate primaries. He considered a multivariate Newton-Raphson iterative scheme for revealing the corresponding basins of convergence on $(x-y)$ and $(y-z)$ planes. He also demonstrated how the oblateness coefficient $A$ influenced the positions of out-of-plane equilibrium points.

Our aim in this paper is to study the existence and stability of the out-of-plane equilibrium points under an oblate primary and a luminous-triaxial secondary in the ER3BP with application to the binary systems DP-Leonis and PSR 1903-0327.

This article is organized in 7 sections as follows: the first section is the introduction; the equations of motion are given in Section 2; Section 3 contains the surface of zero-velocity curves $(\mathrm{ZVC})$ in the $(\xi-\zeta)$ plane; the positions and stability of the out-of-plane equilibrium points are treated in Sections 4 and 5, respectively; Section 6 contains numerical application, while Section 7 is the discussion which concludes the study.

\section{Equations of Motion}

The equations of motion of a test particle in the ER3BP with a bigger oblate primary and a radiating-triaxial secondary in dimensionless-pulsating (rotating) coordinate $\operatorname{system}(\xi, \eta, \zeta)$ are given in $[5,6]$ as follows:

$$
\begin{aligned}
\xi^{\prime \prime}-2 \eta^{\prime} & =\Omega_{\xi} \\
\eta^{\prime \prime}+2 \xi^{\prime} & =\Omega_{\eta} \\
\zeta^{\prime \prime} & =\Omega_{\zeta}
\end{aligned}
$$

where the prime in equations (1) denotes differentiation with respect to the eccentric anomaly $E$ ( $E$ is an angular parameter that defines the position of a body that is moving along the elliptic Keplerian orbit; see [5] for more details) and $\Omega$ is the potential-like function defined by

$$
\begin{aligned}
\Omega & =\left(1-e^{2}\right)^{-1 / 2}\left[\frac{\xi^{2}+\eta^{2}}{2}+\frac{1}{n^{2}}\left\{\frac{(1-\mu)}{r_{1}}+\frac{\mu q_{2}}{r_{2}}\right.\right. \\
& +\frac{(1-\mu) A}{2 r_{1}^{3}}-\frac{3(1-\mu) \zeta^{2} A}{2 r_{1}^{5}}+\frac{\mu q_{2}\left(2 \sigma_{1}-\sigma_{2}\right)}{2 r_{2}^{3}} \\
& \left.\left.-\frac{3 \mu q_{2}\left(2 \sigma_{1}-\sigma_{2}\right) \zeta^{2}}{2 r_{2}^{5}}\right\}\right] .
\end{aligned}
$$

With $r_{1}^{2}=(\xi+\mu)^{2}+\eta^{2}+\zeta^{2}$ and $r_{2}^{2}=(\xi+\mu-1)^{2}+\eta^{2}+\zeta^{2}$ as the distances of the third body $m_{3}$ from the primary body $m_{1}$ and secondary body $m_{2}$, respectively. Also $e$ and $a$ are the eccentricity and semimajor axis of the orbit of the primaries, respectively, $n$ is the perturbed mean motion of the primaries, while $q_{2}$ is the radiation pressure factor of the secondary body which is given by $F_{g}-F_{p}=F_{g}\left(1-F_{p} / F_{g}\right)=q_{2} F_{g}$, where $q_{2}=1-F_{p} / F_{g}$ such that $0<1-q_{2}=\delta \ll 1$ (Radzievskii $[14])$, and $F_{g}$ and $F_{p}$ are, respectively, the gravitational and radiation pressure forces. Additionally, $A=\left(a_{1}^{2}-a_{3}^{2}\right) / 5 R^{2} \ll$ $1, \sigma_{1}=\left(b_{1}^{2}-b_{3}^{2}\right) / 5 R^{2} \ll 1$, and $\sigma_{2}=\left(b_{2}^{2}-b_{3}^{2}\right) / 5 R^{2} \ll 1$, where $a_{1}$ and $a_{3}$ are, respectively, the equatorial and polar radii of the primary body, $b_{1}, b_{2}$, and $b_{3}$ are the lengths of the semiaxes of the secondary body, and $R$ is the dimensional distance between the primaries.

2.1. Jacobi Constant. Equations (1) admit the Jacobi constant:

$$
2 \Omega-\left(\xi^{\prime 2}+\eta^{\prime 2}+\zeta^{\prime 2}\right)=C
$$

where $C$ is the constant of integration known as Jacobi constant.

2.2. Derivation of the Perturbed Mean Motion. In the case of elliptic orbit, the distance between the primaries is $r=a(1-$ $\left.e^{2}\right) /(1+e \cos v)$ and the mean distance between them is given by

$$
\frac{1}{2 \pi} \int_{0}^{2 \pi} r d \nu=\frac{a\left(1-e^{2}\right)}{\left(1+e^{2}\right)^{1 / 2}} .
$$

The orbits of $m_{1}$ and $m_{2}$ with respect to the centre of mass, with semimajor axes $a_{1}=a m_{2}$ and $a_{2}=a m_{1}$, respectively, have the same eccentricity with (see Szebehely [1]):

Their equations are given by

$$
\begin{aligned}
& \frac{n^{2} a m_{1}\left(1-e^{2}\right)}{\left(1+e^{2}\right)^{1 / 2}}=k^{2} m_{2}\left(1+\frac{3 A}{2}+\frac{3\left(2 \sigma_{1}-\sigma_{2}\right)}{2}\right), \\
& \frac{n^{2} a m_{2}\left(1-e^{2}\right)}{\left(1+e^{2}\right)^{1 / 2}}=k^{2} m_{1}\left(1+\frac{3 A}{2}+\frac{3\left(2 \sigma_{1}-\sigma_{2}\right)}{2}\right),
\end{aligned}
$$

where $k$ is the Gaussian constant of gravitation. 


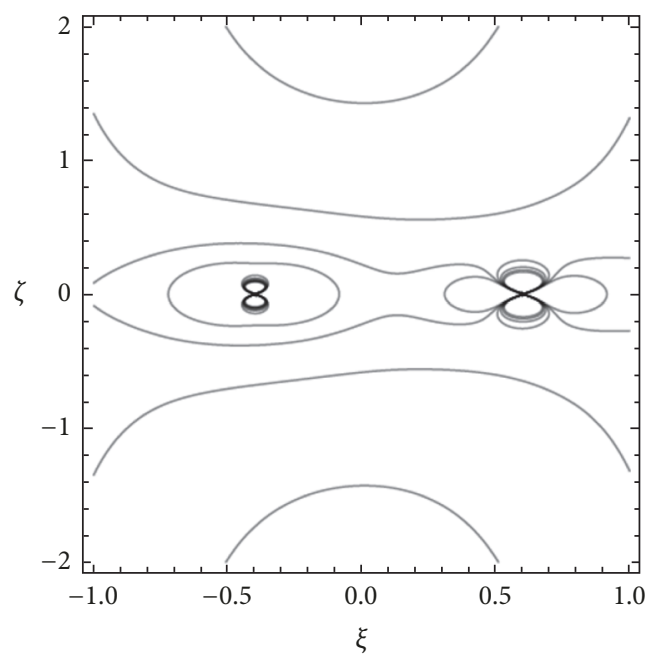

(a)

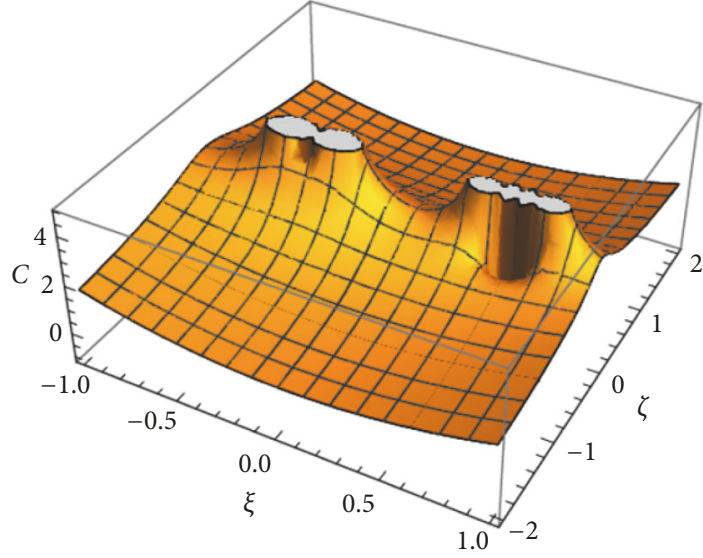

(b)

FIGURE 1: Zero-velocity curves in the $(\xi-\zeta)$ plane of PSR $1903+0327$ for $A=0.01, a=0.95, \sigma_{1}=0.03$ and $\sigma_{2}=0.015$.

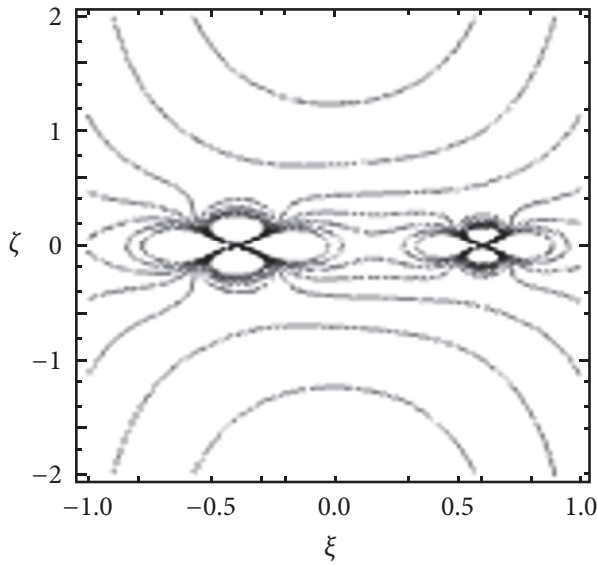

(a)

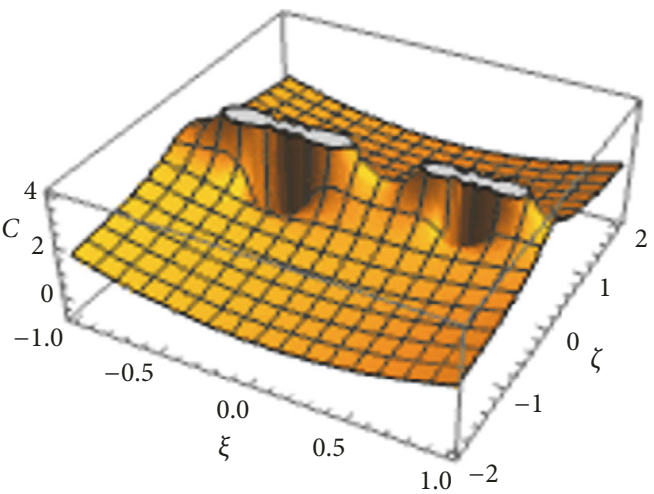

(b)

Figure 2: Zero-velocity curves in the $(\xi-\zeta)$ plane of PSR $1903+0327$ for $A=0.1, a=0.95, \sigma_{1}=0.03$ and $\sigma_{2}=0.015$.

Adding equations (5) together we have

$$
n^{2}=\frac{k^{2}\left(1+e^{2}\right)^{1 / 2}}{a\left(1-e^{2}\right)}\left(1+\frac{3 A}{2}+\frac{3\left(2 \sigma_{1}-\sigma_{2}\right)}{2}\right) .
$$

Here, we adopt that the sum of the masses of the primaries and the distances between them as the units of mass and length. We choose the unit of time such as making $k^{2}=1$. Hence,

$$
n^{2}=\frac{\left(1+e^{2}\right)^{1 / 2}}{a\left(1-e^{2}\right)}\left(1+\frac{3 A}{2}+\frac{3\left(2 \sigma_{1}-\sigma_{2}\right)}{2}\right) .
$$

Making the simplification of the above $n^{2}$, by rejecting the second and higher order terms of $e^{2}$, we get

$$
n^{2}=\frac{1}{a}\left(1+\frac{3 e^{2}}{2}+\frac{3 A}{2}+\frac{3\left(2 \sigma_{1}-\sigma_{2}\right)}{2}\right) .
$$

\section{Surface of Zero-Velocity Curves (ZVC) in the $(\xi-\zeta)$ Plane}

We introduce the ZVC as our quantitative method of understanding information about the motion near out-of-plane equilibrium points without actually solving the differential equations. The potentials of the principal bodies under consideration in this study have significant effects on the existence and the locations of the out-of-plane equilibrium points and, also, on the structure of the boundary between region of possible motion and forbidden region. So, we present the ZVC in the $(\xi-\zeta)$ plane in this section for the binary systems under consideration (i.e., PSR 1903+0327 and DP-Leonis. See Section 6 for more details of the binaries). In Figures 1-4, we demonstrate the possible topologies of the curves for PSR 1903+0327, while Figures 5-10 demonstrate the possible topologies of the curves for DP-Leonis. For clarity, in each case we show the $3 \mathrm{D}$ (i.e., the right frame 


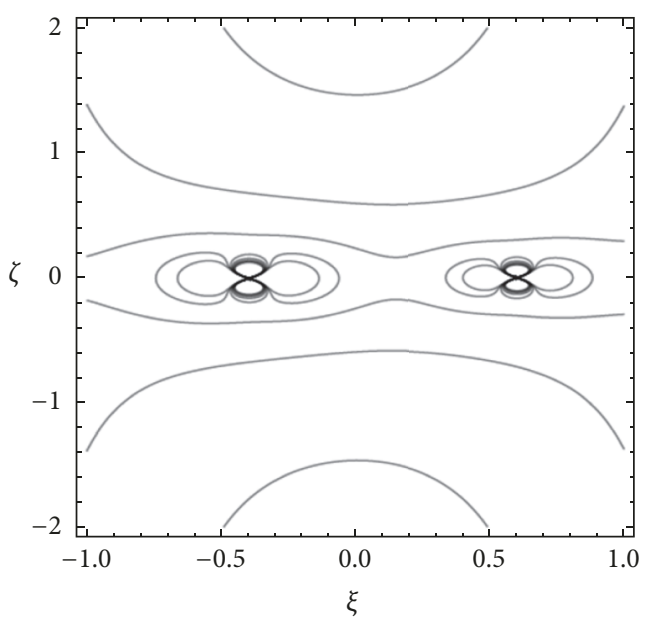

(a)

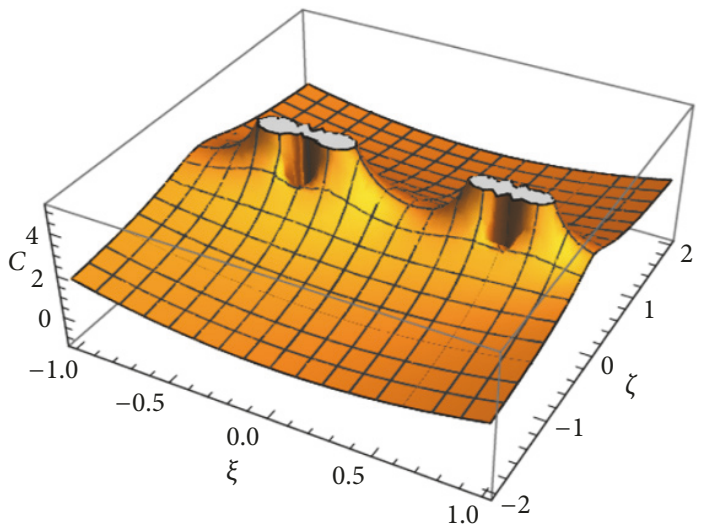

(b)

Figure 3: Zero-velocity curves in the $(\xi-\zeta)$ plane of PSR $1903+0327$ for $A=0.02, a=0.95, \sigma_{1}=0.01$ and $\sigma_{2}=0.005$.

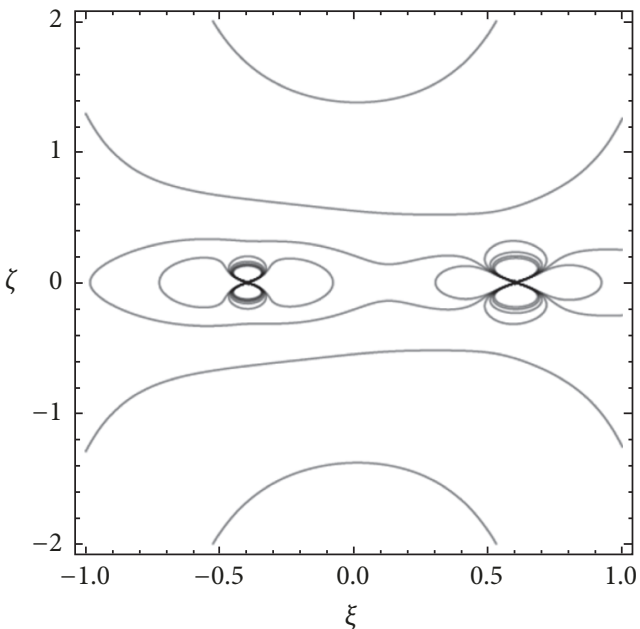

(a)

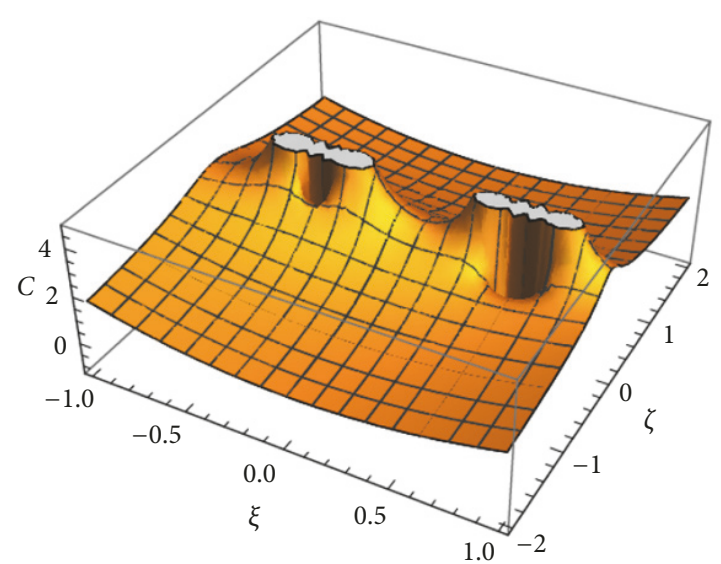

(b)

FIGURE 4: Zero-velocity curves in the $(\xi-\zeta)$ plane of PSR $1903+0327$ for $A=0.02, a=0.95, \sigma_{1}=0.01$ and $\sigma_{2}=0.005$.

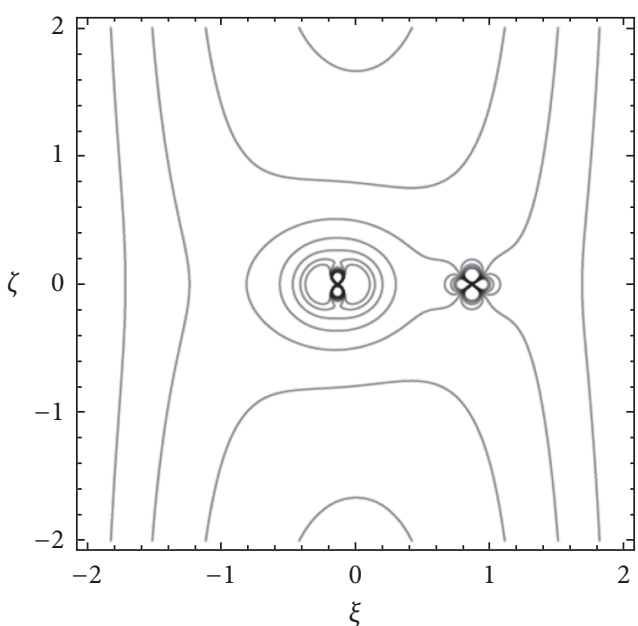

(a)

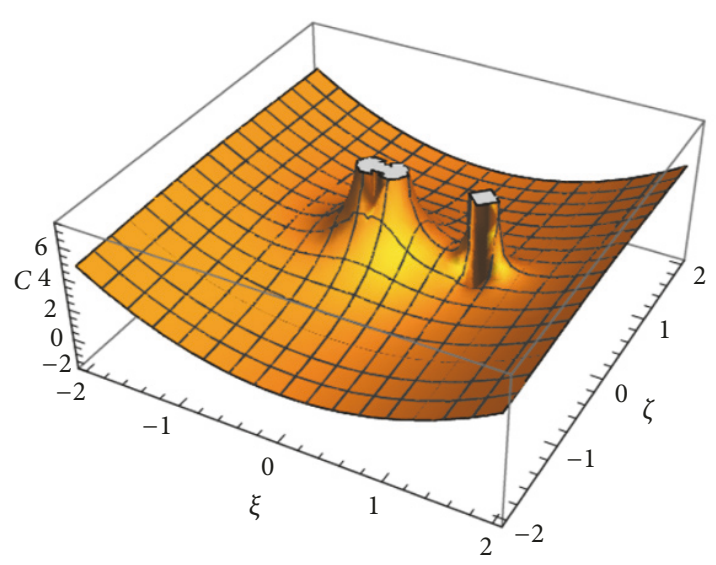

(b)

Figure 5: Zero-velocity curves in the $(\xi-\zeta)$ plane of DP-Leonis for $A=0.01, e=0.2, a=0.95, \sigma_{1}=0.03$ and $\sigma_{2}=0.015$. 


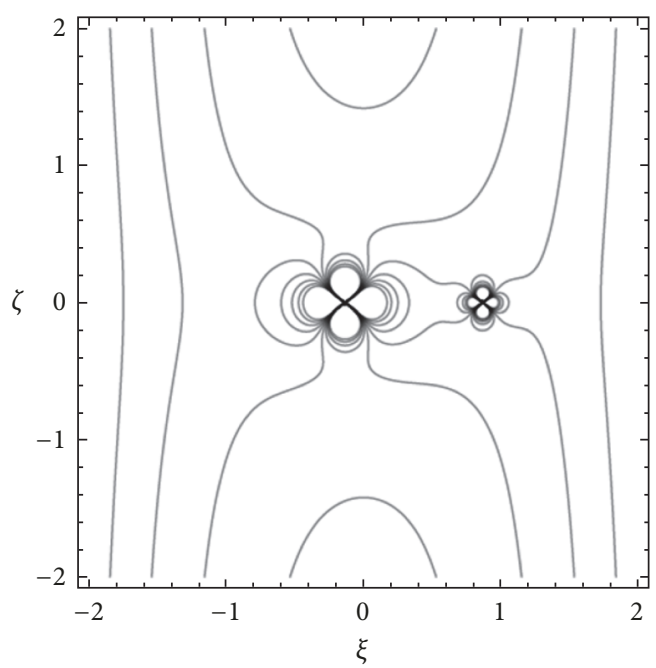

(a)

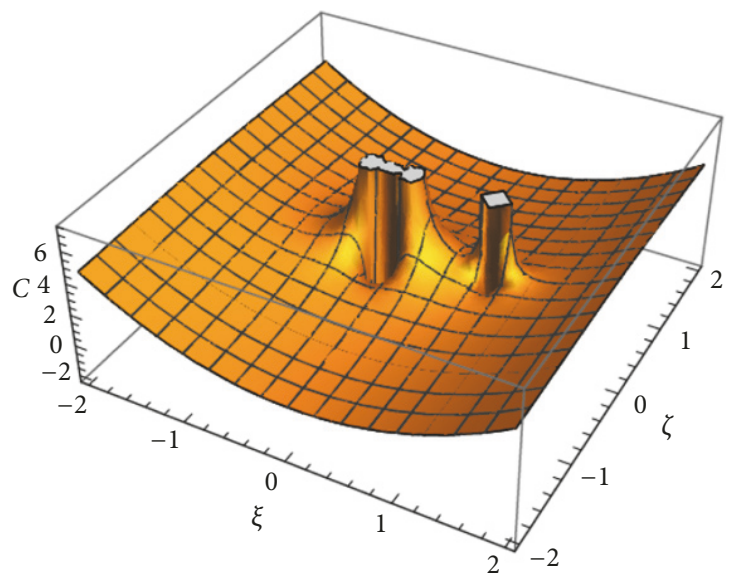

(b)

FIgURE 6: Zero-velocity curves in the $(\xi-\zeta)$ plane of DP-Leonis for $A=0.1, e=0.2, a=0.95, \sigma_{1}=0.03$ and $\sigma_{2}=0.015$.

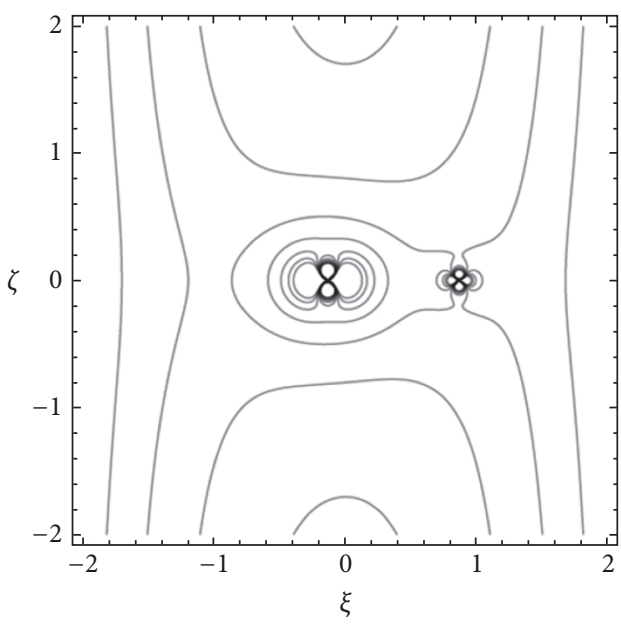

(a)

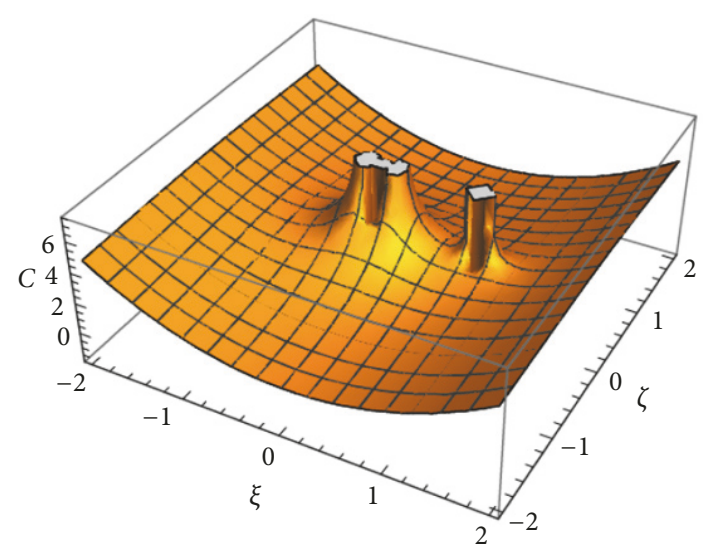

(b)

Figure 7: Zero-velocity curves in the $(\xi-\zeta)$ plane of DP-Leonis for $A=0.02, e=0.2, a=0.95, \sigma_{1}=0.01$ and $\sigma_{2}=0.005$.

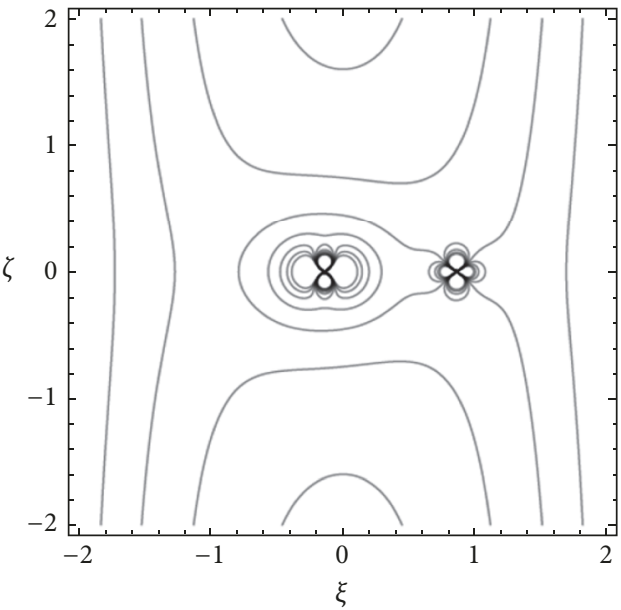

(a)

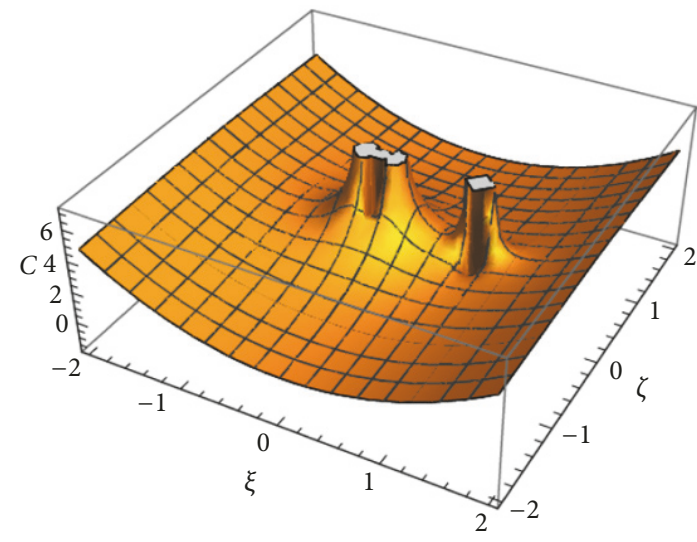

(b)

Figure 8: Zero-velocity curves in the $(\xi-\zeta)$ plane of DP-Leonis for $A=0.02, e=0.2, a=0.95, \sigma_{1}=0.04$ and $\sigma_{2}=0.02$. 


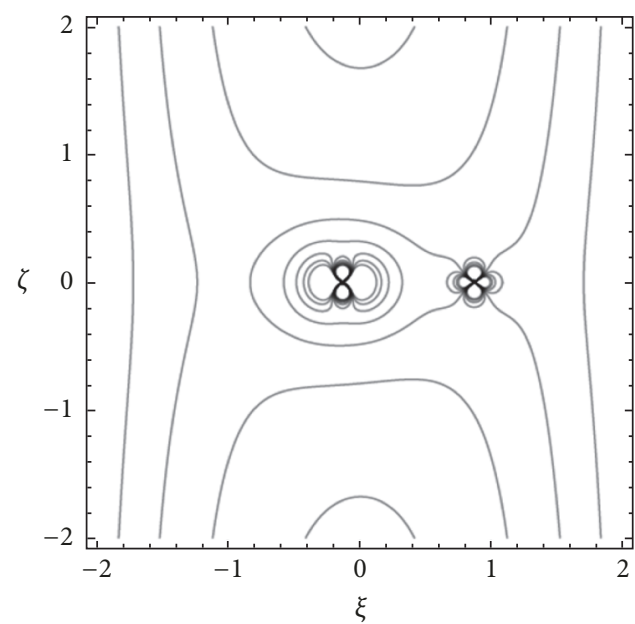

(a)

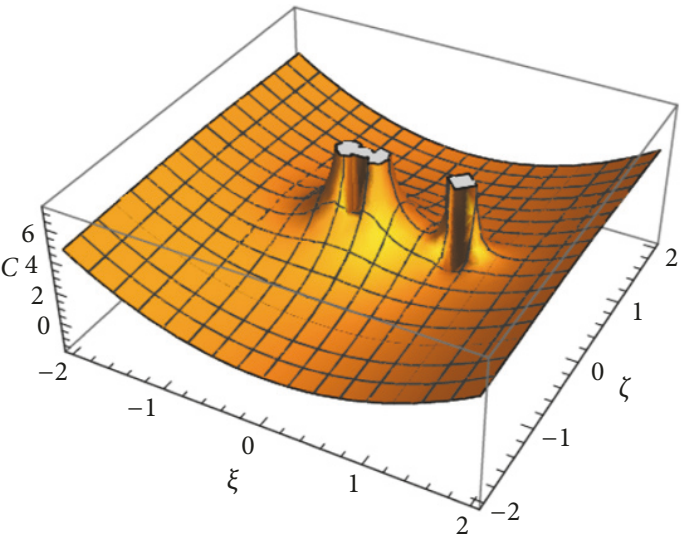

(b)

Figure 9: Zero-velocity curves in the $(\xi-\zeta)$ plane of DP-Leonis for $A=0.02, e=0.1, a=0.95, \sigma_{1}=0.03$ and $\sigma_{2}=0.015$.

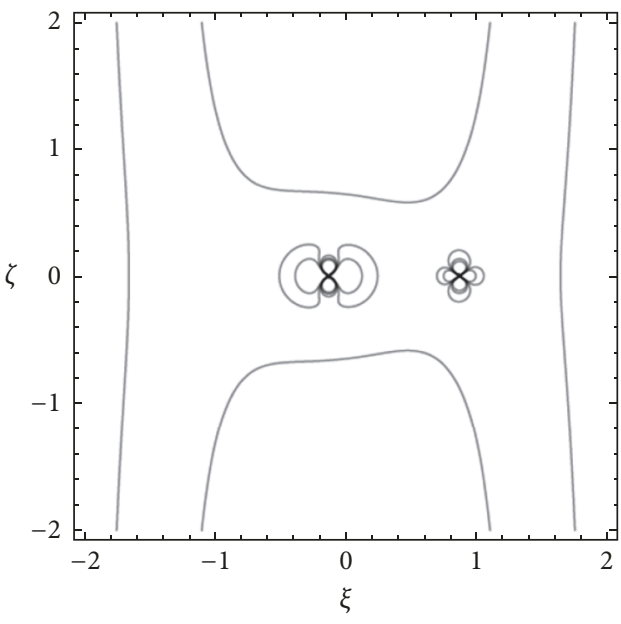

(a)

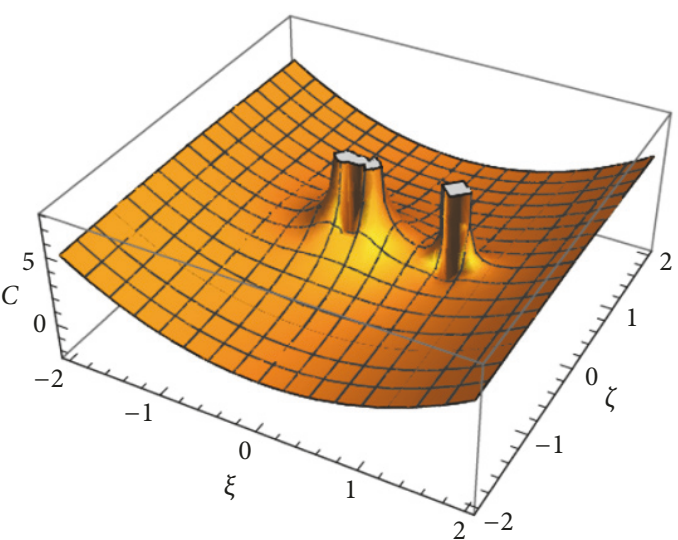

(b)

Figure 10: Zero-velocity curves in the $(\xi-\zeta)$ plane of DP-Leonis for $A=0.02, e=0.5, a=0.95, \sigma_{1}=0.03$ and $\sigma_{2}=0.015$.

in each row) representation of the curves corresponding to the left frame in each row. In the case of PSR 1903+0327, Figures 1 and 2 and Figures 3 and 4 show the topologies of the ZVC for varying the values of $A$ and $\left(\sigma_{1} \& \sigma_{2}\right)$, respectively, when keeping remaining parameters constant. For the system DP-Leonis, Figures 5 and 6, Figures 7 and 8, and Figures 9 and 10 show the topologies of the ZVC for varying values of $A,\left(\sigma_{1} \& \sigma_{2}\right)$, and $e$, respectively, when keeping remaining parameters constant.

\section{Positions of the Out-of-Plane Equilibrium Points}

The solutions of the system $\Omega_{\xi}=\Omega_{\eta}=\Omega_{\zeta}=0$ when $(\xi \neq$ $0, \eta=0$ and $\zeta \neq 0$ ) represent the positions of the out-ofplane equilibrium points $\left(\xi_{0}, 0, \zeta_{0}\right)$; that is,

$$
\Omega_{\xi}=\left(1-e^{2}\right)^{-1 / 2}\left[\xi-\frac{1}{n^{2}}\left\{\frac{(1-\mu)(\xi+\mu)}{r_{1}^{3}}\right.\right.
$$

$$
\begin{aligned}
& +\frac{\mu q_{2}(\xi+\mu-1)}{r_{2}^{3}}+\frac{3(1-\mu)(\xi+\mu) A}{2 r_{1}^{5}} \\
& +\frac{3 \mu q_{2}(\xi+\mu-1)\left(2 \sigma_{1}-\sigma_{2}\right)}{2 r_{2}^{5}} \\
& -\frac{15(1-\mu)(\xi+\mu) \zeta^{2} A}{2 r_{1}^{7}} \\
& \left.\left.-\frac{15 \mu q_{2}(\xi+\mu-1)\left(2 \sigma_{1}-\sigma_{2}\right) \zeta^{2}}{2 r_{2}^{7}}\right\}\right]=0,
\end{aligned}
$$

$$
\begin{aligned}
\Omega_{\eta} & =\left(1-e^{2}\right)^{-1 / 2} \eta\left[1-\frac{1}{n^{2}}\left\{\frac{(1-\mu)}{r_{1}^{3}}+\frac{\mu q_{2}}{r_{2}^{3}}\right.\right. \\
& +\frac{3(1-\mu) A}{2 r_{1}^{5}}+\frac{3 \mu q_{2}\left(4 \sigma_{1}-3 \sigma_{2}\right)}{2 r_{2}^{5}}
\end{aligned}
$$




$$
\begin{aligned}
& \left.\left.-\frac{15(1-\mu) A \zeta^{2}}{2 r_{1}^{7}}-\frac{15 \mu q_{2}\left(2 \sigma_{1}-\sigma_{2}\right) \zeta^{2}}{2 r_{2}^{7}}\right\}\right]=0, \\
\Omega_{\zeta} & =\left(1-e^{2}\right)^{-1 / 2}\left[-\frac{\zeta}{n^{2}}\left\{\frac{(1-\mu)}{r_{1}^{3}}+\frac{\mu q_{2}}{r_{2}^{3}}\right.\right. \\
& +\frac{9(1-\mu) A}{2 r_{1}^{5}}+\frac{9 \mu q_{2}\left(2 \sigma_{1}-\sigma_{2}\right)}{2 r_{2}^{5}}-\frac{15(1-\mu) A \zeta^{2}}{2 r_{1}^{7}} \\
& \left.\left.-\frac{15 \mu q_{2}\left(2 \sigma_{1}-\sigma_{2}\right) \zeta^{2}}{2 r_{2}^{7}}\right\}\right]=0 .
\end{aligned}
$$

From (10) with $\eta=0$ we have

$$
\begin{aligned}
n^{2}- & \frac{(1-\mu)}{r_{1}^{3}}-\frac{\mu q_{2}}{r_{2}^{3}}-\frac{3(1-\mu) A}{2 r_{1}^{5}} \\
& -\frac{3 \mu q_{2}\left(4 \sigma_{1}-3 \sigma_{2}\right)}{2 r_{2}^{5}}+\frac{15(1-\mu) A \zeta^{2}}{2 r_{1}^{7}} \\
& +\frac{15 \mu q_{2}\left(2 \sigma_{1}-\sigma_{2}\right) \zeta^{2}}{2 r_{2}^{7}} \neq 0 .
\end{aligned}
$$

Also, from (11) with $\zeta \neq 0$ we have

$$
\begin{aligned}
& \frac{(1-\mu)}{r_{1}^{3}}+\frac{\mu q_{2}}{r_{2}^{3}}+\frac{9(1-\mu) A}{2 r_{1}^{5}}+\frac{9 \mu q_{2}\left(2 \sigma_{1}-\sigma_{2}\right)}{2 r_{2}^{5}} \\
& -\frac{15(1-\mu) A \zeta^{2}}{2 r_{1}^{7}}-\frac{15 \mu q_{2}\left(2 \sigma_{1}-\sigma_{2}\right) \zeta^{2}}{2 r_{2}^{7}}=0 .
\end{aligned}
$$

Here, we let $Q_{1}=(1-\mu)$ and $Q_{2}=\mu q_{2}$; then (13) becomes

$$
\begin{aligned}
\frac{Q_{1}}{r_{1}^{3}}+ & \frac{Q_{2}}{r_{2}^{3}}+\frac{9 Q_{1} A}{2 r_{1}^{5}}+\frac{9 Q_{2}\left(2 \sigma_{1}-\sigma_{2}\right)}{2 r_{2}^{5}}-\frac{15 Q_{1} A \zeta^{2}}{2 r_{1}^{7}} \\
& -\frac{15 Q_{2}\left(2 \sigma_{1}-\sigma_{2}\right) \zeta^{2}}{2 r_{2}^{7}}=0
\end{aligned}
$$

while (9) becomes

$$
\begin{aligned}
n^{2} \xi & -\frac{Q_{1}(\xi+\mu)}{r_{1}^{3}}-\frac{Q_{2}(\xi+\mu-1)}{r_{2}^{3}}-\frac{3 Q_{1}(\xi+\mu) A}{2 r_{1}^{5}} \\
& -\frac{3 Q_{2}(\xi+\mu-1)\left(2 \sigma_{1}-\sigma_{2}\right)}{2 r_{2}^{5}} \\
& +\frac{15 Q_{1}(\xi+\mu) \zeta^{2} A}{2 r_{1}^{7}} \\
& +\frac{15 Q_{2}(\xi+\mu-1)\left(2 \sigma_{1}-\sigma_{2}\right) \zeta^{2}}{2 r_{2}^{7}}=0 .
\end{aligned}
$$

This implies that

$$
\begin{aligned}
\xi\{ & 1-\frac{1}{n^{2}}\left(\frac{Q_{1}}{r_{1}^{3}}+\frac{Q_{2}}{r_{2}^{3}}+\frac{3 Q_{1} A}{2 r_{1}^{5}}-\frac{15 Q_{1} \zeta^{2} A}{2 r_{1}^{7}}\right. \\
& \left.+\frac{3 Q_{2}\left(2 \sigma_{1}-\sigma_{2}\right)}{2 r_{2}^{5}}-\frac{15 Q_{2}\left(2 \sigma_{1}-\sigma_{2}\right) \zeta^{2}}{2 r_{2}^{7}}\right) \\
& -\frac{\mu}{n^{2}}\left(\frac{Q_{1}}{r_{1}^{3}}+\frac{Q_{2}}{r_{2}^{3}}+\frac{3 Q_{1} A}{2 r_{1}^{5}}-\frac{15 Q_{1} \zeta^{2} A}{2 r_{1}^{7}}\right. \\
& \left.+\frac{3 Q_{2}\left(2 \sigma_{1}-\sigma_{2}\right)}{2 r_{2}^{5}}-\frac{15 Q_{2}\left(2 \sigma_{1}-\sigma_{2}\right) \zeta^{2}}{2 r_{2}^{7}}\right) \\
& +\frac{1}{n^{2}}\left(\frac{Q_{2}}{r_{2}^{3}}+\frac{3 Q_{2}\left(2 \sigma_{1}-\sigma_{2}\right)}{2 r_{2}^{5}}\right. \\
& \left.-\frac{15 Q_{2}\left(2 \sigma_{1}-\sigma_{2}\right) \zeta^{2}}{2 r_{2}^{7}}\right)=0 .
\end{aligned}
$$

Making use of (14) in (16), we have

$$
\xi=\frac{Q_{1} / r_{1}^{3}+9 Q_{1} A / 2 r_{1}^{5}+3 Q_{2} Q_{1}\left(2 \sigma_{1}-\sigma_{2}\right) / r_{2}^{5}-15 Q_{1} A \zeta^{2} / 2 r_{1}^{7}-3 \mu Q_{1} A / r_{1}^{5}}{n^{2}+3 Q_{1} A / 2 r_{1}^{5}+3 Q_{2}\left(2 \sigma_{1}-\sigma_{2}\right) / 2 r_{2}^{5}} .
$$

From (14), we have

$$
\begin{aligned}
\zeta^{2} & =\frac{2 r_{1}^{7} r_{2}^{7}}{15 Q_{1} A r_{2}^{7}+15 Q_{2}\left(2 \sigma_{1}-\sigma_{2}\right) r_{1}^{7}}\left\{\frac{Q_{1}}{r_{1}^{3}}+\frac{9 Q_{1} A}{2 r_{1}^{5}}\right. \\
& \left.+\frac{Q_{2}}{r_{2}^{3}}+\frac{9 Q_{2}\left(2 \sigma_{1}-\sigma_{2}\right)}{2 r_{2}^{5}}\right\} .
\end{aligned}
$$

Here, we use $\xi_{0}=(1-\mu)$ and $\zeta_{0}=\sqrt{3} \sqrt{A}$ as initial approximation. Using Wolfam Mathematica 10.3 (software package) we are able to obtain the positions of a pair of points $\left(\xi_{0}, 0, \pm \zeta_{0}\right)$ corresponding to out-of-plane equilibrium points
$L_{6,7}$ which can be approximated in the forms of power series to the third order terms in $A$ from (17) and (18) as follows:

$$
\begin{aligned}
\xi_{0} & =(1-\mu) \\
& -\frac{3 \sqrt{3}(1-\mu)\left\{2(1-a)+3 e^{2}+3\left(2 \sigma_{1}-\sigma_{2}\right)\right\} A^{5 / 2}}{2\left(a \mu q_{2}\left(2 \sigma_{1}-\sigma_{2}\right)\right)} \\
& +0(A)^{7 / 2} \\
\zeta_{0} & =\frac{3 \sqrt{5 A}}{5}+\frac{\sqrt{5} A^{3 / 2}}{5\left(2 \sigma_{1}-\sigma_{2}\right)}-\frac{\sqrt{5} A^{5 / 2}}{30\left(2 \sigma_{1}-\sigma_{2}\right)^{2}}
\end{aligned}
$$




$$
+\frac{3 \sqrt{15}(\mu-1) A^{3}}{5 \mu q_{2}\left(2 \sigma_{1}-\sigma_{2}\right)^{2}}+0(A)^{7 / 2}
$$

The effects of the parameters involved in the positions of the $1^{\text {st }}$ family of out-of-plane equilibrium points are shown graphically for the binary systems PSR 1903+0327 and DPLeonis, respectively, in Figures 11-14. Figures 11 and 13 show the positions of $L_{6,7}$ in the $(\xi-\zeta)$ plane as a function of oblateness $A$ in the interval $[0,0.1]$, keeping the remaining parameters constant for the binary systems PSR 1903+0327 and DP-Leonis, respectively, while Figures 12 and 14 show the positions of $L_{6,7}$ in the $(\xi-\zeta)$ plane as a function of triaxiality $\sigma_{1} \& \sigma_{2}$ in the intervals [0.01, 0.02] and [0.005, 0.01], respectively, keeping remaining parameters constant for the binary systems PSR 1903+0327 and DP-Leonis, respectively.

\section{Linear Stability of the Out-of-Plane Equilibrium Points}

In order to study the stability of motion near the out-of-plane equilibrium point, we adopt the characteristic equation of the system as in $[4,21]$, given as follows:

$$
\begin{aligned}
& \lambda^{6}+\left(4-\Omega_{\xi \xi}^{0}-\Omega_{\eta \eta}^{0}-\Omega_{\zeta \zeta}^{0}\right) \lambda^{4} \\
& +\left(\Omega_{\eta \eta}^{0} \Omega_{\zeta \zeta}^{0}+\Omega_{\xi \xi}^{0} \Omega_{\zeta \zeta}^{0}+\Omega_{\xi \xi}^{0} \Omega_{\eta \eta}^{0}-4 \Omega_{\zeta \zeta}^{0}-\left(\Omega_{\xi \zeta}^{0}\right)^{2}\right) \lambda^{2} \\
& -\left(\Omega_{\xi \xi}^{0} \Omega_{\eta \eta}^{0} \Omega_{\zeta \zeta}^{0}-\left(\Omega_{\xi \zeta}^{0}\right)^{2} \Omega_{\eta \eta}^{0}\right)=0
\end{aligned}
$$

where the superscript 0 denotes that the partial derivatives are evaluated at the out-of-plane point $\left(\xi_{0}, 0, \zeta_{0}\right)$.

So, at the out-of-plane equilibrium points we have

$$
\begin{aligned}
\Omega_{\xi \xi}^{0} & =\left(1-e^{2}\right)^{-1 / 2}\left[1+\frac{1}{n^{2}}\left\{\frac{3 Q_{1}\left(\xi_{0}+\mu\right)^{2}}{r_{10}^{5}}-\frac{Q_{1}}{r_{10}^{3}}\right.\right. \\
& +\frac{3 Q_{2}\left(\xi_{0}+\mu-1\right)^{2}}{r_{20}^{5}}-\frac{Q_{2}}{r_{20}^{3}}+\frac{15 Q_{1}\left(\xi_{0}+\mu\right)^{2} A}{2 r_{10}^{7}} \\
& -\frac{3 Q_{1} A}{2 r_{10}^{5}}-\frac{105 Q_{1}\left(\xi_{0}+\mu\right)^{2} \zeta_{0}^{2} A}{2 r_{10}^{9}}+\frac{15 Q_{1} \zeta_{0}^{2} A}{2 r_{10}^{7}} \\
& +\frac{15 Q_{2}\left(\xi_{0}+\mu-1\right)^{2}\left(2 \sigma_{1}-\sigma_{2}\right)}{2 r_{20}^{7}} \\
& -\frac{3 Q_{2}\left(2 \sigma_{1}-\sigma_{2}\right)}{2 r_{20}^{5}} \\
& -\frac{105 Q_{2}\left(\xi_{0}+\mu-1\right)^{2}\left(2 \sigma_{1}-\sigma_{2}\right) \zeta_{0}^{2}}{2 r_{20}^{9}} \\
+ & \left.\frac{15 Q_{2}\left(2 \sigma_{1}-\sigma_{2}\right) \zeta_{0}^{2}}{2 r_{20}^{7}}\right\}
\end{aligned}
$$

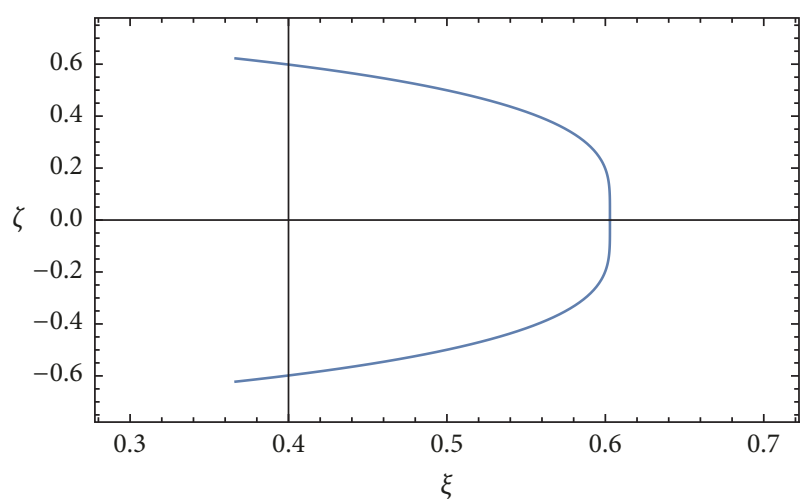

Figure 11: Positions of $L_{6,7}$ in the $(\xi-\zeta)$ plane as a function of oblateness $A$ in the interval $[0,0.1]$ of PSR $1903+0327$ for $a=$ $0.95, \sigma_{1}=0.03$ and $\sigma_{2}=0.015$.

$$
\begin{aligned}
& \Omega_{\eta \eta}^{0}=\left(1-e^{2}\right)^{-1 / 2}\left[1-\frac{1}{n^{2}}\left\{\frac{Q_{1}}{r_{10}^{3}}+\frac{Q_{2}}{r_{20}^{3}}+\frac{3 Q_{1} A}{2 r_{10}^{5}}\right.\right. \\
& -\frac{15 Q_{1} \zeta_{0}^{2} A}{2 r_{10}^{7}}+\frac{3 Q_{2}\left(4 \sigma_{1}-3 \sigma_{2}\right)}{2 r_{20}^{5}} \\
& \left.\left.-\frac{15 Q_{2}\left(2 \sigma_{1}-\sigma_{2}\right) \zeta_{0}^{2}}{2 r_{20}^{7}}\right\}\right] \\
& \Omega_{\zeta \zeta}^{0}=\left(1-e^{2}\right)^{-1 / 2}\left[\frac { 1 } { n ^ { 2 } } \left\{\frac{3 Q_{1} \zeta_{0}^{2}}{r_{10}^{5}}-\frac{Q_{1}}{r_{10}^{3}}+\frac{3 Q_{2} \zeta_{0}^{2}}{r_{20}^{5}}-\frac{Q_{2}}{r_{20}^{3}}\right.\right. \\
& +\frac{45 Q_{1} \zeta_{0}^{2} A}{2 r_{10}^{7}}-\frac{9 Q_{1} A}{2 r_{10}^{5}}-\frac{105 Q_{1} \zeta_{0}^{4} A}{2 r_{10}^{9}} \\
& +\frac{45 Q_{2}\left(2 \sigma_{1}-\sigma_{2}\right) \zeta_{0}^{2}}{2 r_{20}^{7}}-\frac{9 Q_{2}\left(2 \sigma_{1}-\sigma_{2}\right)}{2 r_{20}^{5}} \\
& \left.\left.-\frac{105 Q_{2}\left(2 \sigma_{1}-\sigma_{2}\right) \zeta_{0}^{4}}{2 r_{20}^{9}}\right\}\right] \\
& \Omega_{\xi \zeta}^{0}=\left(1-e^{2}\right)^{-1 / 2}\left[\frac { 3 \zeta _ { 0 } } { n ^ { 2 } } \left\{\frac{3 Q_{1}\left(\xi_{0}+\mu\right)}{r_{10}^{5}}\right.\right. \\
& +\frac{Q_{2}\left(\xi_{0}+\mu-1\right)}{r_{20}^{5}}+\frac{15 Q_{1}\left(\xi_{0}+\mu\right) A}{2 r_{10}^{7}} \\
& -\frac{35 Q_{1}\left(\xi_{0}+\mu\right) \zeta_{0}^{2} A}{2 r_{10}^{9}} \\
& +\frac{15 Q_{2}\left(\xi_{0}+\mu-1\right)\left(2 \sigma_{1}-\sigma_{2}\right)}{2 r_{20}^{7}} \\
& \left.\left.-\frac{35 Q_{2}\left(\xi_{0}+\mu-1\right)\left(2 \sigma_{1}-\sigma_{2}\right) \zeta_{0}^{2}}{2 r_{20}^{9}}\right\}\right]
\end{aligned}
$$

\section{Numerical Application}

In this section, we apply the data (barrowed from NASA ADS and [7]) of the binaries PSR 1903+0327 and DP-Leonis 
TABLE 1: Relevant numerical data.

\begin{tabular}{lcccccc}
\hline \multirow{2}{*}{ Binary System } & \multicolumn{2}{c}{ Masses $\left(M_{\odot}\right)$} & Luminosity & Mass ratio & Radiation pressure & Eccentricity \\
& $m_{1}$ & $m_{2}$ & $\left(L_{\odot}\right)$ & $(\mu)$ & $\left(q_{2}\right)$ & 0.997581 \\
\hline PSR 1903+0327 & 1.6700 & 1.1000 & 2.5000 & 0.397112 & 0.437 \\
\hline DP-Leonis & 0.600 & 0.090 & 0.00449 & 0.130435 & 0.999947 \\
\hline
\end{tabular}

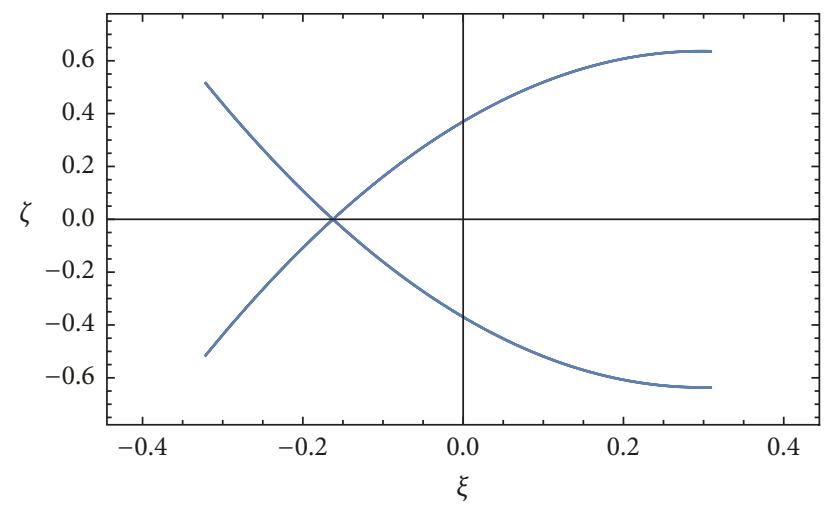

Figure 12: Positions of $L_{6,7}$ in the $(\xi-\zeta)$ plane as a function of triaxiality $\sigma_{1}$ and $\sigma_{2}$ in the interval $[0.01,0.02]$ and $[0.005,0.01]$, respectively, of PSR $1903+0327$ for $a=0.95, A=0.1$.

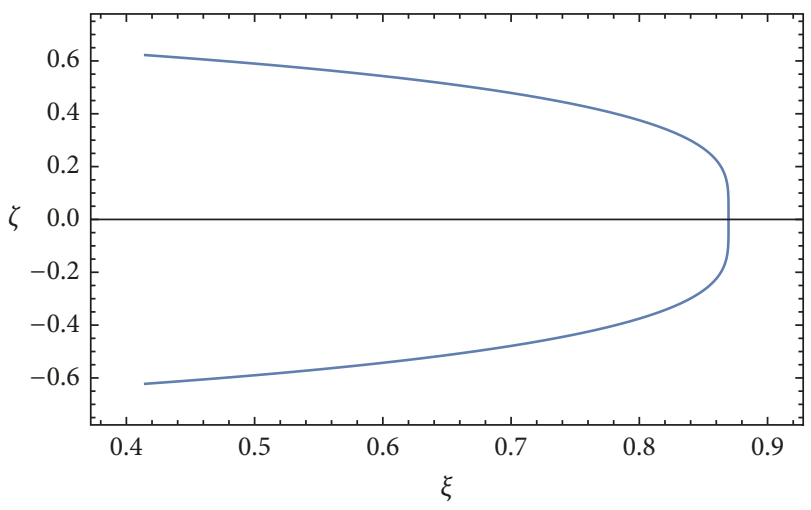

Figure 13: Positions of $L_{6,7}$ in the $(\xi-\zeta)$ plane as a function of oblateness $A$ in the interval $[0,0.1]$ of DP-Leonis for $a=0.95, e=$ $0.2, \sigma_{1}=0.03$ and $\sigma_{2}=0.015$.

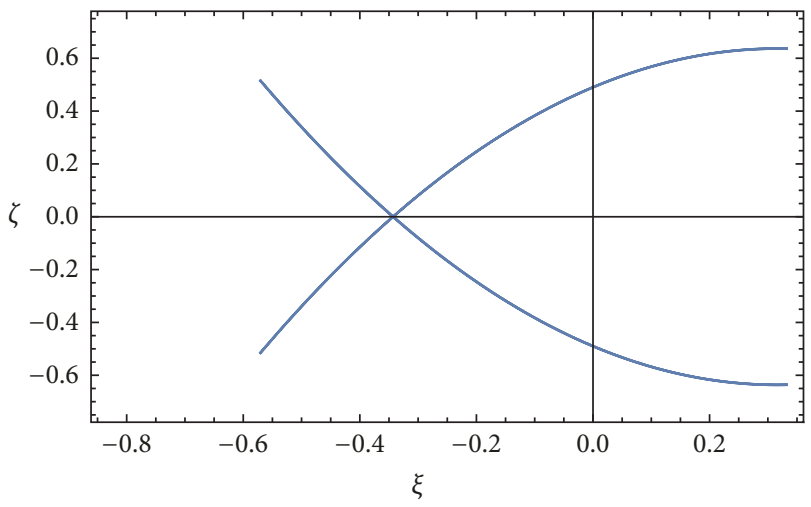

Figure 14: Positions of $L_{6,7}$ in the $(\xi-\zeta)$ plane as a function of triaxiality $\sigma_{1}$ and $\sigma_{2}$ in the interval $[0.01,0.02]$ and $[0.005,0.01]$, respectively, of DP-Leonis for $a=0.95, e=0.2$ and $A=0.1$.
(Table 1) to the analytical results obtained in the previous sections under the assumption that the primary body is an oblate spheroid while the secondary body is a luminoustriaxial body both moving in elliptic Keplerian orbits. The positions of the out-of-plane equilibrium points (Equation (19)) together with the roots of the characteristic equation (Equation (20)) are computed (for some assumed values of the potential of the system under consideration) in Tables 2-4 for PSR 1903+0327 and in Tables 5-7 for DP-Leonis with the help of the Wolfram Mathematica 10.3.

\section{Discussion}

The equations of motion of a third body under the influence of an oblate primary and a radiating-triaxial secondary moving in an elliptic Keplerian orbits are described by the governing equations (1).

It is seen in Figures 1-4 that the regions above and below the principal bodies are allowed to motion, while the regions between the principal bodies, the regions at the left of the primary body, the regions at the right of the secondary body, and the small regions nearer to the principal bodies are not allowed to motion. Also, a close study of Figures 5-9 reveals that all the regions are allowed to motion except the regions between the principal bodies and the small regions nearer to the principal bodies for each figure (i.e., Figures 5-9), while in Figure 10 all the regions are allowed to motion except the small regions nearer to the principal bodies due to the high value of eccentricity $e$. It is also seen that the topologies of the ZVC for the binary systems PSR 1903+0327 and DP-Leonis differ. This happens due to the effects of their mass ratio $\mu$. We also observed that the topology of the ZVC may change depending on the energy of the third body.

Equations (19) give the pair of points $\left(\xi_{0}, 0, \pm \zeta_{0}\right)$ which correspond to the $1^{\text {st }}$ family of the out-of-plane equilibrium points, $L_{6,7}$. It can be seen from (19) that the point $\xi_{0}$ is affected by the oblateness of the primary body, radiation and triaxiality of the secondary body, semimajor axis, and eccentricity of the orbits of the principal bodies. Meanwhile, the point $\pm \zeta_{0}$ is independent of the semimajor axis and eccentricity of the orbits of the principal bodies; it is only affected by the oblateness of the primary body and radiation pressure as well as triaxiality of the secondary body. This is similar to $[5,21]$ in which the point $\pm \zeta_{0}$ is independent of the semimajor axis and eccentricity of the orbits of principal bodies.

It is seen in Figures 12 and 14 that there exist values of triaxiality corresponding to the out-of-plane equilibrium points which coincides with the values corresponding to the collinear equilibrium points in particular, the inner 
TABLE 2: Effects of oblateness of PSR 1903+0327 on the locations and stability of out-of-plane points for $a=0.95, e=0.437, \sigma_{1}=$ 0.03 and $\sigma_{2}=0.015$.

\begin{tabular}{lcccccc}
\hline$A$ & $\xi$ & $\pm \zeta$ & $\lambda_{1,2}$ & $\lambda_{3,4}$ & $\lambda_{5,6}$ & Remark \\
\hline 0.01 & 0.602141 & 0.143655 & \pm 39.12118 & $\pm 98.95687 i$ & \pm 35.94876 & unstable \\
\hline 0.02 & 0.598661 & 0.215135 & $-12.78173 \pm 0.6731746 i$ & $\pm 36.07696 i$ & $12.78173 \pm 0.6731746 i$ & unstable \\
\hline 0.03 & 0.591240 & 0.276159 & $-6.249728 \pm 0.8922171 i$ & $\pm 19.32210 i$ & $6.249728 \pm 0.8922171 i$ & unstable \\
\hline 0.04 & 0.578977 & 0.331025 & $-3.497018 \pm 0.9379422 i$ & $\pm 12.24203 i$ & $3.497018 \pm 0.9379422 i$ & unstable \\
\hline 0.05 & 0.561117 & 0.380711 & $-2.031369 \pm 0.9503968 i$ & $\pm 8.546200 i$ & $2.031369 \pm 0.9503968 i$ & unstable \\
\hline
\end{tabular}

TABLE 3: Effects of eccentricity of PSR $1903+0327$ on the locations and stability of out-of-plane points for $a=0.95, A=0.02, \sigma_{1}=$ 0.03 and $\sigma_{2}=0.015$.

\begin{tabular}{lcccccc}
\hline$e$ & $\xi$ & $\pm \zeta$ & $\lambda_{1,2}$ & $\lambda_{3,4}$ & $\lambda_{5,6}$ & Remark \\
\hline 0.1 & 0.602643 & 0.143655 & \pm 37.44600 & $\pm 94.78687 i$ & \pm 34.45998 & unstable \\
\hline 0.2 & 0.602560 & 0.143655 & \pm 37.72920 & $\pm 95.49115 i$ & \pm 34.71129 & unstable \\
\hline 0.3 & 0.602421 & 0.143655 & \pm 38.19624 & $\pm 96.65320 i$ & \pm 35.12607 & unstable \\
\hline 0.4 & 0.602227 & 0.143655 & \pm 38.84000 & $\pm 98.25624 i$ & \pm 35.69849 & unstable \\
\hline 0.5 & 0.601977 & 0.143655 & \pm 39.65119 & $\pm 100.2783 i$ & \pm 36.42090 & unstable \\
\hline
\end{tabular}

TABLE 4: Effects of triaxiality of PSR $1903+0327$ on the locations and stability of out-of-plane points for $a=0.95, e=0.437$ and $A=0.02$.

\begin{tabular}{cccccccc}
\hline$\sigma_{1}$ & $\sigma_{2}$ & $\xi$ & $\pm \zeta$ & $\lambda_{1,2}$ & $\lambda_{3,4}$ & $\lambda_{5,6}$ & Remark \\
\hline 0.01 & 0.005 & 0.591620 & 0.253439 & $-2.546035 \pm 0.771621 i$ & $\pm 14.71489 i$ & $2.546035 \pm 0.771621 i$ & Unstable \\
\hline 0.02 & 0.010 & 0.596901 & 0.226272 & $-8.552855 \pm 0.7998829 i$ & $\pm 26.47024 i$ & $8.552855 \pm 0.7998829 i$ & Unstable \\
\hline 0.03 & 0.015 & 0.598669 & 0.215135 & $-12.78173 \pm 0.6731746 i$ & $\pm 36.07696 i$ & $12.78173 \pm 0.6731746 i$ & Unstable \\
\hline 0.04 & 0.020 & 0.599541 & 0.209176 & $-16.19443 \pm 0.4955597 i$ & $\pm 44.06055 i$ & $16.19443 \pm 0.4955597 i$ & Unstable \\
\hline 0.05 & 0.025 & 0.600069 & 0.205475 & $-19.07191 \pm 0.183883 i$ & $\pm 50.88468 i$ & $19.07191 \pm 0.183883 i$ & Unstable \\
\hline
\end{tabular}

TABLE 5: Effects of oblateness of DP-Leonis on the locations and stability of out-of-plane points for $a=0.95, e=0.2, \sigma_{1}=0.03$ and $\sigma_{2}=$ 0.015 .

\begin{tabular}{lcccccc}
\hline$A$ & $\xi$ & $\pm \zeta$ & $\lambda_{1,2}$ & $\lambda_{3,4}$ & $\lambda_{5,6}$ & Remark \\
\hline 0.01 & 0.868127 & 0.143390 & \pm 21.23742 & \pm 20.52800 & $\pm 55.03901 i$ & Unstable \\
\hline 0.02 & 0.861428 & 0.213009 & $-7.258800 \pm 0.8860236 i$ & $\pm 20.43774 i$ & $7.258800 \pm 0.8860236 i$ & Unstable \\
\hline 0.03 & 0.847143 & 0.268985 & $-3.688747 \pm 0.9403290 i$ & $\pm 11.31166 i$ & $3.688747 \pm 0.9403290 i$ & Unstable \\
\hline 0.04 & 0.823537 & 0.314020 & $-2.172492 \pm 0.9468649 i$ & $\pm 7.487427 i$ & $2.172492 \pm 0.9468649 i$ & Unstable \\
\hline 0.05 & 0.789157 & 0.347500 & $-1.312145 \pm 0.9376641 i$ & $\pm 5.479935 i$ & $1.312145 \pm 0.9376641 i$ & Unstable \\
\hline
\end{tabular}

TABLE 6: Effects of eccentricity of DP-Leonis on the locations and stability of out-of-plane points for $a=0.95, A=0.02, \sigma_{1}=0.03$ and $\sigma_{2}=$ 0.015 .

\begin{tabular}{ccccccc}
\hline$e$ & $\xi$ & $\pm \zeta$ & $\lambda_{1,2}$ & $\lambda_{3,4}$ & $\lambda_{5,6}$ & Remark \\
\hline 0.1 & 0.863491 & 0.213009 & $-7.219975 \pm 0.8852772 i$ & $\pm 20.31470 i$ & $7.219975 \pm 0.8852772 i$ & Unstable \\
\hline 0.2 & 0.861428 & 0.213009 & $-7.258800 \pm 0.8860236 i$ & $\pm 20.43774 i$ & $7.258800 \pm 0.8860236 i$ & Unstable \\
\hline 0.3 & 0.857990 & 0.213009 & $-7.311630 \pm 0.8892137 i$ & $\pm 20.62122 i$ & $7.311630 \pm 0.8892137 i$ & Unstable \\
\hline 0.4 & 0.853177 & 0.213009 & $-7.360223 \pm 0.8975893 i$ & $\pm 20.83215 i$ & $7.360223 \pm 0.8975893 i$ & Unstable \\
\hline 0.5 & 0.846988 & 0.213009 & $-7.378476 \pm 0.9140821 i$ & $\pm 21.02381 i$ & $7.378476 \pm 0.9140821 i$ & Unstable \\
\hline
\end{tabular}

TABLE 7: Effects of triaxiality of DP-Leonis on the locations and stability of out-of-plane points for $a=0.95, e=0.2$ and $A=0.02$.

\begin{tabular}{cccccccc}
\hline$\sigma_{1}$ & $\sigma_{2}$ & $\xi$ & $\pm \zeta$ & $\lambda_{1,2}$ & $\lambda_{3,4}$ & $\lambda_{5,6}$ & Remark \\
\hline 0.01 & 0.005 & 0.851343 & 0.247062 & $-1.61690 \pm 0.8535960 i$ & $\pm 8.628061 i$ & $1.61690 \pm 0.8535960 i$ & Unstable \\
\hline 0.02 & 0.010 & 0.858907 & 0.223084 & $-4.92729 \pm 0.9 .117207 i$ & $\pm 15.14238 i$ & $4.92729 \pm 0.9 .117207 i$ & Unstable \\
\hline 0.03 & 0.015 & 0.861428 & 0.213009 & $-7.25880 \pm 0.8860236 i$ & $\pm 20.43774 i$ & $7.25880 \pm 0.8860236 i$ & Unstable \\
\hline 0.04 & 0.020 & 0.862689 & 0.207582 & $-9.136514 \pm 0.852032 i$ & $\pm 24.82868 i$ & $9.136514 \pm 0.852032 i$ & Unstable \\
\hline 0.05 & 0.025 & 0.863445 & 0.204200 & $-10.71929 \pm 0.8148062 i$ & $\pm 28.57866 i$ & $10.71929 \pm 0.8148062 i$ & Unstable \\
\hline
\end{tabular}


TABLE 8: Effects of mass ratio $\mu$ on the locations and stability of out-of-plane points for $a=0.95, e=0.2, q_{2}=0.9999, A=0.08, \sigma_{1}=$ 0.03 and $\sigma_{2}=0.015$.

\begin{tabular}{|c|c|c|c|c|c|c|}
\hline$\mu$ & $\xi_{0}$ & $\pm \zeta_{0}$ & $\lambda_{1,2}$ & $\lambda_{3,4}$ & $\lambda_{5,6}$ & Remark \\
\hline 0.32 & 0.597001 & 0.481528 & $\pm 0.5279522 i$ & $\pm 1.326220 i$ & $\pm 4.025670 i$ & Stable \\
\hline 0.33 & 0.590700 & 0.484032 & $\pm 0.5278752 i$ & $\pm 1.329252 i$ & $\pm 4.058162 i$ & Stable \\
\hline 0.34 & 0.584181 & 0.486388 & $\pm 0.5259979 i$ & $\pm 1.333737 i$ & $\pm 4.089879 i$ & Stable \\
\hline 0.35 & 0.577463 & 0.488610 & $\pm 0.5226098 i$ & $\pm 1.339435 i$ & $\pm 4.120928 i$ & Stable \\
\hline 0.36 & 0.570563 & 0.490709 & $\pm 0.5179634 i$ & $\pm 1.346133 i$ & $\pm 4.151399 i$ & Stable \\
\hline 0.37 & 0.563495 & 0.492694 & $\pm 0.5122757 i$ & $\pm 1.353646 i$ & $\pm 4.181367 i$ & Stable \\
\hline 0.38 & 0.556273 & 0.494575 & $\pm 0.5057316 i$ & $\pm 1.361814 i$ & $\pm 4.210892 i$ & Stable \\
\hline 0.39 & 0.548909 & 0.496359 & $\pm 0.4984878 i$ & $\pm 1.370503 i$ & $\pm 4.240027 i$ & Stable \\
\hline 0.40 & 0.541413 & 0.498054 & $\pm 0.4906756 i$ & $\pm 1.379600 i$ & $\pm 4.268812 i$ & Stable \\
\hline 0.41 & 0.533794 & 0.499666 & $\pm 0.4824047 i$ & $\pm 1.389008 i$ & $\pm 4.297285 i$ & Stable \\
\hline 0.42 & 0.526062 & 0.501202 & $\pm 0.4737663 i$ & $\pm 1.398650 i$ & $\pm 4.325475 i$ & Stable \\
\hline 0.43 & 0.518225 & 0.502666 & $\pm 0.4648359 i$ & $\pm 1.408461 i$ & $\pm 4.353408 i$ & Stable \\
\hline 0.44 & 0.510290 & 0.504064 & $\pm 0.4556760 i$ & $\pm 1.418386 i$ & $\pm 4.381104 i$ & Stable \\
\hline 0.45 & 0.502262 & 0.505399 & $\pm 0.4463377 i$ & $\pm 1.428382 i$ & $\pm 4.408583 i$ & Stable \\
\hline 0.46 & 0.494149 & 0.506676 & $\pm 0.4368632 i$ & $\pm 1.438414 i$ & $\pm 4.435859 i$ & Stable \\
\hline 0.47 & 0.485956 & 0.507900 & $\pm 0.4272867 i$ & $\pm 1.448451 i$ & $\pm 4.462947 i$ & Stable \\
\hline 0.48 & 0.477687 & 0.509072 & $\pm 0.4176362 i$ & $\pm 1.458471 i$ & $\pm 4.489856 i$ & Stable \\
\hline 0.49 & 0.469348 & 0.510196 & $\pm 0.4079342 i$ & $\pm 1.468455 i$ & $\pm 4.516598 i$ & Stable \\
\hline 0.50 & 0.460942 & 0.511275 & $\pm 0.3981986 i$ & $\pm 1.478387 i$ & $\pm 4.543180 i$ & Stable \\
\hline
\end{tabular}

collinear equilibrium points $\left(L_{2}\right)$ for the binary systems PSR $1903+0327$, and DP-Leonis, respectively.

The numerical computations of the roots of characteristic Equation (20), given in Tables 2-7, reveal the existence of at least a positive real root and complex root with positive part for each particular set of values. This means that the motion of an infinitesimal mass near $L_{6,7}$ is unbounded. Hence, the topologies of the ZVC and the nature of the computed roots of the characteristic equation (20) for the binary systems PSR $1903+0327$ and DP-Leonis confirm the instability of the outof-plane equilibrium points which agrees with $[5,14,17,20-$ 22], while the characteristic roots given in Table 8 reveal that all the roots are purely imaginary. This means that the motion of an infinitesimal mass near $L_{6,7}$ is stable. The stability of the out-of-plane equilibrium points resulted from the value of mass ratio $\mu,(0.32 \leq \mu \leq 0.5)$, and the small value of eccentricity.

\section{Data Availability}

The data used to support the findings of this study are included within the article.

\section{Conflicts of Interest}

The authors declare that there are no conflicts of interest regarding the publication of this paper.

\section{References}

[1] V. Szebehely, Theory of Orbits, vol. 1, Academic Press, New York, 1967.
[2] J. Singh and A. Umar, "Application of binary pulsars to axisymmetric bodies in the elliptic R3BP," Astrophysics and Space Science, vol. 348, pp. 393-402, 2012.

[3] V. Kumar and R. K. Choudhry, "Nonlinear stability of the triangular libration points for the photo gravitational elliptic restricted problem of three bodies," Celestial Mechanics and Dynamical Astronomy, vol. 48, no. 2, pp. 299-317, 1990.

[4] R. K. Sharma, Z. A. Taqvi, and K. B. Bhatnagar, "Existence and stability of libration points in the restricted three-body problem when the primaries are triaxial rigid bodies," Celestial Mechanics \& Dynamical Astronomy, vol. 79, no. 2, pp. 119-133, 2001.

[5] J. Singh and A. Umar, "Motion in the photogravitational elliptic restricted three-body problem under an oblate primary," The Astronomical Journal, vol. 143, no. 1, pp. 109-130, 2012.

[6] A. Umar and A. A. Hussain, "Motion in the ER3BP with an oblate primary and a triaxial stellar companion," Astrophysics and Space Science, vol. 361, no. 10, pp. 344-354, 2016.

[7] A. A. Hussain, A. Umar, and J. Singh, "Investigation of the stability of a test particle in the vicinity of collinear points with the additional influence of an oblate primary and a triaxialstellar companion in the frame of ER3BP," International Frontier Science Letters, vol. 13, no. 1, pp. 12-27, 2018.

[8] A. Narayan and S. Amit, "Pulsating different curves of zero velocity around triangular equilibrium points in elliptical restricted three-body problem," Journal of Mathematics, vol. 1, Article ID 936859, 8 pages, 2013.

[9] E. I. Abouelmagd, "The effect of photogravitational force and oblateness in the perturbed restricted three-body problem," Astrophysics and Space Science, vol. 346, no. 1, pp. 51-69, 2013.

[10] B. Nakone and A. A. Hussain, "Collinear Equilibrium points in the relativistic $\mathrm{r} 3 \mathrm{bp}$ when the bigger primary is a triaxial rigid body," International Frontier Science Letters, vol. 11, no. 1, pp. 4556, 2017. 
[11] E. E. Zotos, "Comparing the fractal basins of attraction in the hill problem with oblateness and radiation," Astrophysics and Space Science, vol. 362, no. 190, 2017.

[12] E. E. Zotos, "Basins of convergence of equilibrium points in the pseudo-newtonian planar circular restricted three-body problem," Astrophysics and Space Science, vol. 362, no. 195, 2017.

[13] T. J. Kalvouridis and I. T. Kalvouridis, "Formation and evolution of newton's regions of convergence in some problems of celestial mechanics," Monografías de la Real Academia de Ciencias de Zaragoza, vol. 35, pp. 53-71, 2011.

[14] V. V. Radzievskii, "The restricted problem of three-bodies taking account of light pressure," The Astronomical Journal, vol. 27, no. 2, pp. 250-256, 1950.

[15] Y. A. Chernikov, "The photogravitational restricted three-body problem," Soviet Astronomy, vol. 14, no. 1, pp. 176-181, 1970.

[16] J. F. Simmons, A. J. McDonald, and J. C. Brown, “The restricted 3-body problem with radiation pressure," Celestial Mechanics, vol. 35, no. 1, pp. 145-187, 1985.

[17] O. Ragos and C. Zagouras, "Periodic solutions about the out of plane equilibrium points in the photogravitational restricted three body problem," Celestial Mechanics, vol. 44, no. 1, pp. 135154, 1988.

[18] P. Oberti and A. Vienne, "An upgraded theory for helene, telesto, and calypso," Astronomy \& Astrophysics, vol. 397, no. 1, pp. 353359, 2003.

[19] Z. Xuentang and Y. Lizhong, "Photogravitationally restricted three-body problem and coplanar libration point," Chinese Physics Letters, vol. 10, no. 1, pp. 61-64, 1993.

[20] C. N. Douskos and V. V. Markellos, "Out-Of-Plane equilibrium points in the restricted three-body problem with oblateness," Astronomy \& Astrophysics, vol. 446, no. 1, pp. 357-360, 2006.

[21] C. Douskos, V. Kalantonis, P. Markellos, and E. Perdios, "On Sitnikov-like motions generating new kinds of $3 \mathrm{~d}$ periodic orbits in the R3BP with prolate primaries," Astrophysics and Space Science, vol. 337, no. 1, pp. 99-106, 2012.

[22] Shankaran, J. P. Sharma, and B. Ishwar, "Out-Of-Plane equilibrium points and stability in the generalised photogravitational restricted three body problem," Astrophysics and Space Science, vol. 332, no. 1, pp. 115-119, 2011.

[23] E. I. Abouelmagd and A. Mostafa, "Out of plane equilibrium points locations and the forbidden movement regions in the restricted three-body problem with variable mass," Astrophysics and Space Science, vol. 357, no. 1, pp. 58-68, 2015.

[24] S. Jagadish and U. Aishetu, "On "out of plane" equilibrium points in the elliptic restricted three-body problem with radiating and oblate primaries," Astrophysics and Space Science, vol. 344, no. 1, pp. 13-19, 2013.

[25] S. Jagadish and T. O. Amuda, "Out-of-Plane equilibrium points in the photogravitational CRTBP with oblateness and P-R drag," International Journal of Astronomy and Astrophysics, vol. 36, no. 1, pp. 291-305, 2015.

[26] A. Chakraborty and A. Narayan, "Influence of poyntingrobertson drag and oblateness on existence and stability of out-of-plane equilibrium points in spatial elliptic restricted three-body problem," Journal of Informatics and Mathematical Sciences, vol. 10, pp. 55-72, 2018.

[27] E. E. Zotos, "On the newton-raphson basins of convergence of the out-of-plane equilibrium points in the copenhagen problem with oblate primaries," International Journal of Non-Linear Mechanics, vol. 103, pp. 93-103, 2018. 

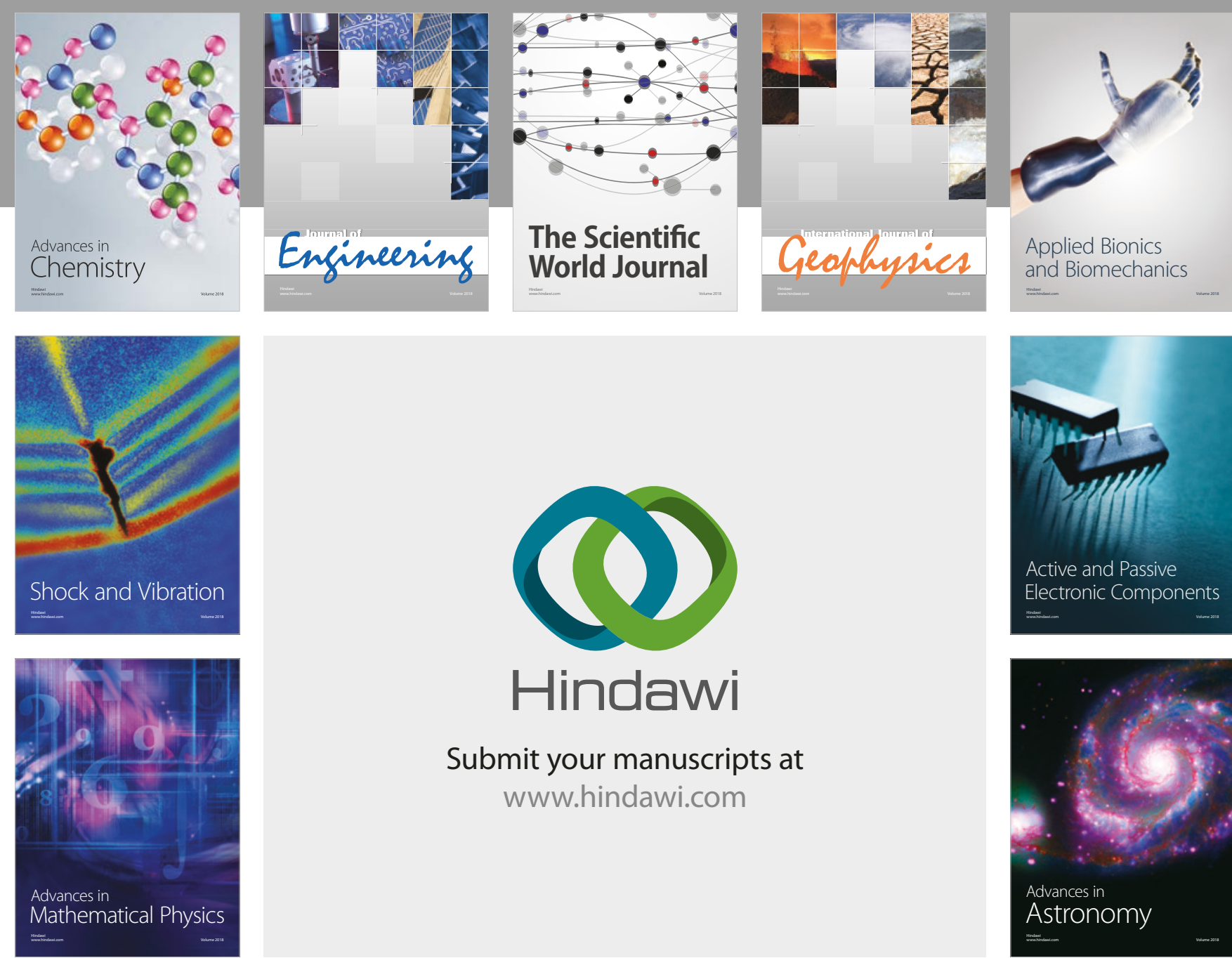

Submit your manuscripts at

www.hindawi.com

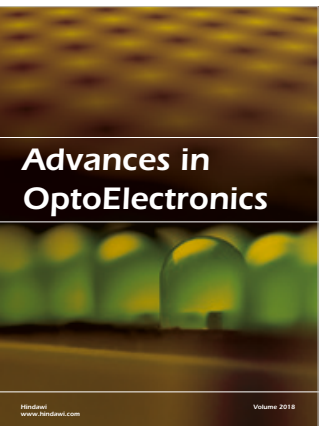

\section{Rotcting Machinery}
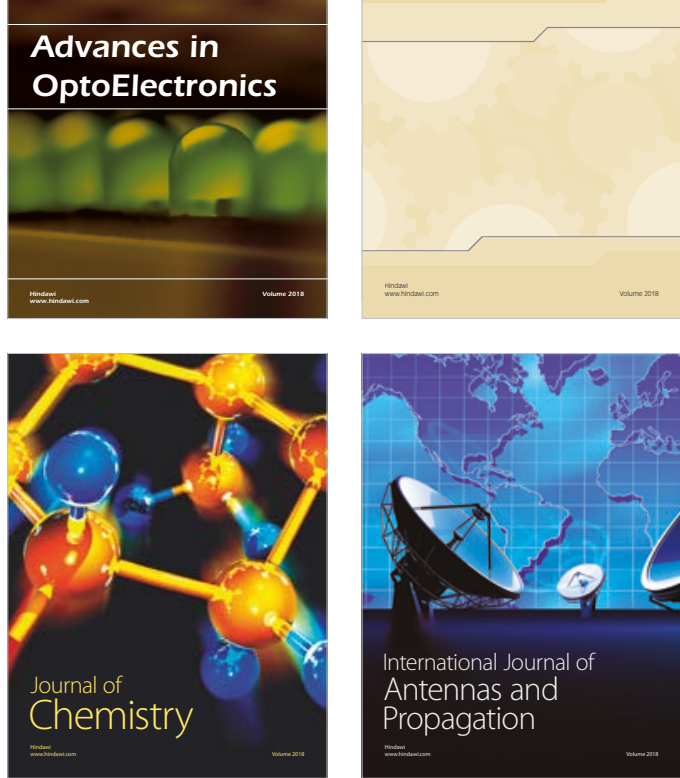

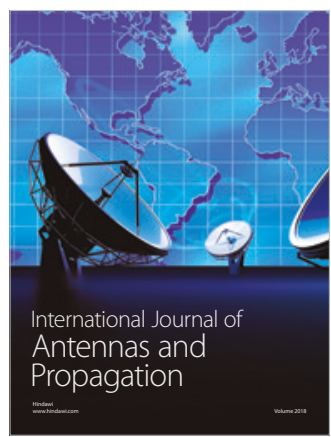

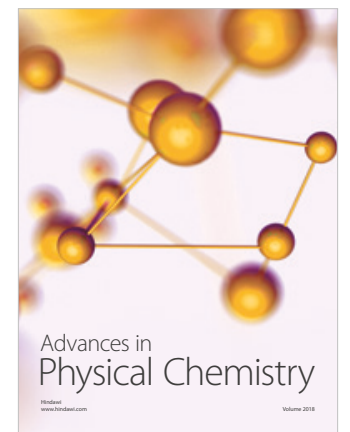

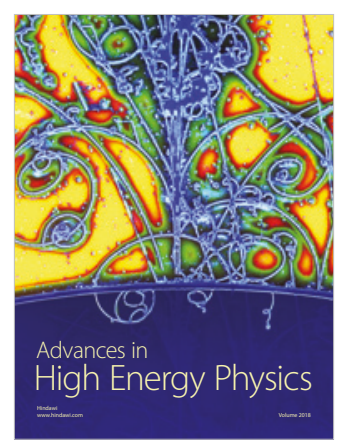

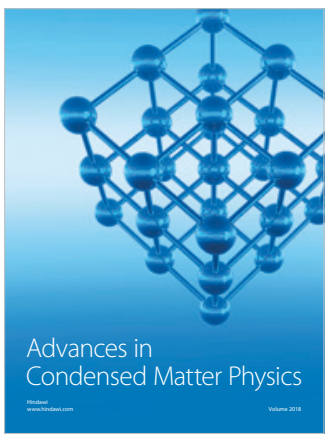

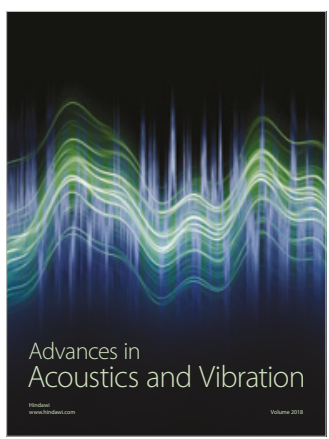

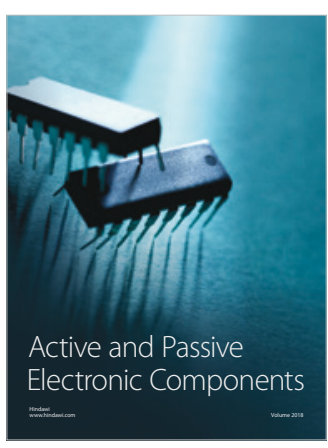
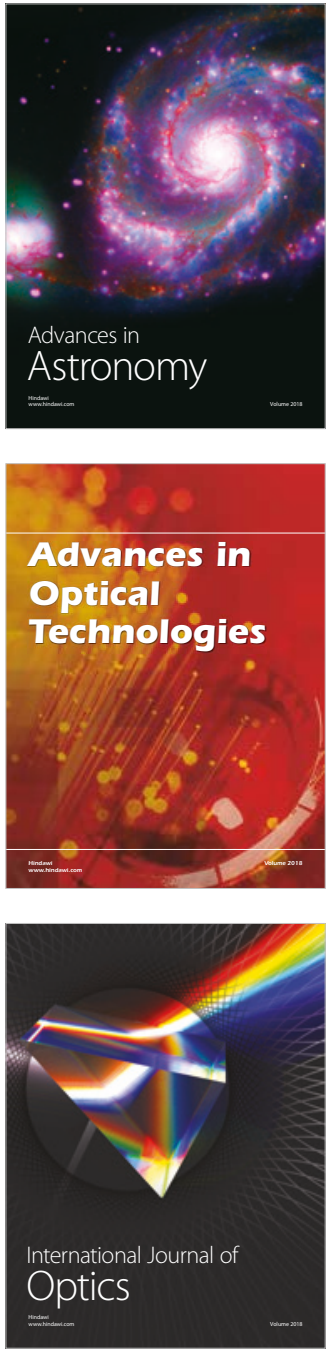